Background Screening for trisomy 21 (T21) in the West Midlands has evolved from double screening in 1995 to combined screening in 2011, with the perception of improved detection amongst the public and providers.

Methods Cases of T21 ( $n=2,608)$ were identified using a regional, population-based, multiple source anomaly register covering a birth cohort of 1,140,866 between 1995 and 2011. Regional data on invasive testing were available for the same period.

Results The total prevalence of T21 increased by approximately $50 \%$ from 18.2 to 27.5 per 10,000 births over the study period. The proportion of cases with a prenatal karyotype was unchanged (annual trend $\chi^{2}=0.21, p=0.65 ; 52.8 \%$ in 1995 and $52.0 \%$ in 2011 . However, karyotyping was taking place earlier in pregnancy; median gestation at procedure 19 weeks (1995) to 13 weeks (2011). TOP rates following prenatal karyotyping were unchanged, and the live birth rate was increased Within the total population (affected and unaffected pregnancies), the rate of amniocentesis and CVS combined for T21 indications decreased by $76 \%$ (4.7\% of births in 1995 to $1.1 \%$ in 2011).

Conclusion The time and effort to develop the T21 screening programme has resulted in safer pregnancies for unaffected cases. Mothers of affected pregnancies have seen no improvement in prenatal diagnosis. The combination of an increasing prevalence of T21 with no change in prenatal diagnosis nor TOP rates means the live birth prevalence continues to rise at the same pace.

\section{PF.06 NEONATAL OUTCOMES FOLLOWING EXTREMELY PRETERM PRELABOUR RUPTURE OF MEMBRANES (EPPROM) IN MULTIPLE PREGNANCY}

doi:10.1136/archdischild-2013-303966.018

S Hirsi-farah, S Kumar. OCCH, London, UK

Aims and Objectives To assess the neonatal outcome of multifetal pregnancies that are complicated by Extremely Preterm Prelabour Rupture of Membranes (EPPROM) under 24 weeks of gestation.

To assess antenatal, and postnatal morbidity for both mothers and babies.

Study design The following is a retrospective observational study of twins and triplets who were referred to the Centre for Fetal Care (CFC) at Queen Charlotte's and Chelsea Hospital (OCCH) who had an antenatal diagnosis of spontaneous EPPROM under completed 24 weeks of gestation. The population were subdivided in to the following three groups: monochorionic, dichorionic, and trichorionic. The scan data base, the obstetrics and the neonatal records of 52 women and their 108 fetuses, were the sources of the information. Our study covered a 10 year period (2002-2012), and then result was analysed

Results The median gestation at PPROM was $22+5$ weeks (range $13-23+6)$. The latency period Median was 10 days (range 1-91 days), while the mean gestational age at delivery was $25+2$ weeks (range 18-37) and the median fetal weight was $630 \mathrm{~g}$ (range $290-3500 \mathrm{~g}$ ), pregnancy loss of $33.3 \%$ and neonatal of $29.63 \%$. The overall survival rate was $36.11 \%$ (39 fetuses).

Conclusion Neonatal survival in EPPROM is very poor (36.11\%), comer in male fetuses who had worse outcome in terms of prematurity complications and perinatal/neonatal survival.

MCDA twins had worse outcome than DCDA, in terms of antenatal complications and survival (36.36\% v $72.97 \%)$.

The optimal management with EPPROM before viability remains controversial and guideline are lacking

\section{PF.07 EXPRESSION OF 2, 3-BISPHOSPHOGLYCERATE MUTASE (BPGM) IN HUMAN PLACENTA}

doi:10.1136/archdischild-2013-303966.019

'MNM Walter, 'SY Chan, ${ }^{2} \mathrm{M}$ Vatish, 'MD Kilby. 'University of Birmingham, Birmingham, UK; ${ }^{2}$ University of Warwick, Warwick, UK
Introduction BPGM is an enzyme in erythrocytes and trophoblasts, which synthesises 2, 3-bisphosphoglycerate (2, 3-BPG), a facilitator of oxygen liberation from haemoglobin. In an insulinlike growth factor II knockout mouse model of intrauterine growth restriction (IUGR), placental BPGM expression is lower than in wild type animals, implicating BPGM in the pathophysiology of IUGR and suggesting a role for 2, 3-BPG in oxygen delivery to the fetus.

Methods Human placental messenger RNA encoding BPGM was quantified by TaqMan RT-PCR. The relative expression of BPGM was assessed a) over the course of pregnancy at 7-11, 12-20, 24-34 weeks of gestation ( $w$ ) and term (total $n=68), b$ ) in IUGR placenta at early (24-34 w, n = 15) and late $(37-39 \mathrm{w}, \mathrm{n}=5)$ gestations and compared with appropriately grown for gestation age (AGA) controls ( $\mathrm{n}=8$ early, $\mathrm{n}=26$ late).

Results BPGM mRNA expression significantly increased with advancing gestation (ANOVA $p<0.001$ ). There was a 6 and 7 -fold increase from $7-11 \mathrm{w}$ to $24-34 \mathrm{w}$ and term respectively, and 3 -fold between $12-20 \mathrm{w}$ and term ( $p<0.05$ for all). There were no statistically significant differences in BPGM mRNA expression between IUGR and AGA placenta in either gestational age group.

Discussion Levels of BPGM increased in a time-dependant manner to term. This may indicate a protective mechanism to avoid oxidative stress damage during the early stages of fetal development, with BPGM expression increasing over time in response to greater oxygen demand from the growing fetus. Placental BPGM expression does not appear to be implicated in the pathogenesis of IUGR in human

\section{PF.08 OUANTITATIVE FIBRONECTIN CAN BE USED FOR EARLIER PREDICTION OF PRETERM BIRTH FROM 18 WEEKS, BUT THE POSITIVE THRESHOLD NEEDS REDEFINING}

doi:10.1136/archdischild-2013-303966.020

NL Hezelgrave, DS Abbott, SK Radford, P Seed, R Tribe, AH Shennan. Division of Women's Health KCL, Women's Health Academic Centre, KHP, London, UK

Introduction Fetal fibronectin (fFN) is an excellent predictor of spontaneous preterm birth (sPTB) and is used qualitatively ( $<50 \mathrm{ng} / \mathrm{ml}$ negative threshold) from 22 weeks gestation. Its value at earlier gestations using a quantitative test (qfFN) is unknown.

Methods A prospective secondary analysis of 431 asymptomatic women at high risk of sPTB, who underwent qfFN testing at 18-21 + 6 weeks. 327 women underwent later testing at $22-26+6$ weeks (acting as their own controls). The end-points were sPTB/ preterm premature rupture of membranes and delivery before 30, 34 and 37 weeks gestation and within 8 weeks of testing.

Results Early qfFN predicted delivery within 8 weeks of testing, $<30,<34$ and $<37$ weeks with receiver operating characteristics (ROC) areas of $0.66(0.54-0.80, \mathrm{p}<0.05), 0.68(0.56-0.79, \mathrm{p}<0.01)$, $0.68(0.58-0.78 \mathrm{p}<0.001)$ and $0.64(0.57-0.72, \mathrm{p}<0.001) .22$-week test prediction was ROC areas of $0.77(0.63-0.91 p<0.001), 0.78$ $(0.61-0.95, \mathrm{p}<0.001)$ and $0.79(0.70-0.89, \mathrm{p}<0.001)$ respectively. A qFFN result of $<10 \mathrm{ng} / \mathrm{ml}$ at earlier gestations had only $1 \%, 2 \%$ and $4.3 \%$ of women delivered within 8 weeks, $<30$ and $<34$ weeks gestation, rising to $6.7 \%, 8.1 \%$ and $14.1 \%$ with values between $10-49.9$ $\mathrm{ng} / \mathrm{ml}$ (all differences statistically significant, $\mathrm{p}=0.03,0.02,0.004$ by Fishers-Exact). The 22 -week test had $1.0 \%, 1.0 \%$ and $2 \%$ respectively, rising to $6.7 \%, 3.3 \%$ and $14 \%$ with values between $10-49.9 \mathrm{ng} / \mathrm{ml}$ ( $\mathrm{p}=0.02,0.23,0.004)$.

Conclusion qfFN is valid for screening for sPTB at 18 weeks, but has inferior predictive value to 22 weeks. Early identification may enable earlier targeted management. A threshold of $<10 \mathrm{ng} / \mathrm{ml}$ is more appropriate than current practise to define low risk at 18 weeks. 